\title{
Assertiveness Expectancies: How Hard People Push Depends on the Consequences They Predict
}

\author{
Daniel R. Ames \\ Columbia University
}

\begin{abstract}
The present article seeks to explain varying levels of assertiveness in interpersonal conflict and negotiations with assertiveness expectancies, idiosyncratic predictions people make about the social and instrumental consequences of assertive behavior. This account complements motivation-based models of assertiveness and competitiveness, suggesting that individuals may possess the same social values (e.g., concern for relationships) but show dramatically different assertiveness due to different assumptions about behavioral consequences. Results clarify the form of assertiveness expectancies, namely that most people assume increasing assertiveness can yield positive social and instrumental benefits up to a point, beyond which benefits decline. However, people vary in how assertive this perceived optimal point is. These individual differences in expectancies are linked in 4 studies to assertiveness, including selfreported assertiveness, rated behavioral preferences in assorted interpersonal conflict scenarios, partner ratings of participants' behavior in a face-to-face dyadic negotiation, and work colleague ratings of participants' assertiveness in the workplace. In each case, the link between expectancies and behavior remained after controlling for values. The results suggest a place for expectancies alongside values in psychological models of interpersonal assertiveness.
\end{abstract}

Keywords: assertiveness, competitiveness, expectancies, conflict, interpersonal schemas

Why, in interpersonal conflicts, do some people come on strong, make bold demands, and concede little ground, whereas others give way, yielding to another person's requests and surrendering their own interests? Over the last generation, the literatures on conflict, negotiation, and social dilemmas have highlighted the importance of values in answering this question, tracing higher levels of assertiveness to proself orientations and competitive conflict styles and lower levels of assertiveness to prosocial orientations and cooperative conflict styles (e.g., Carnevale \& De Dreu, 2006; De Dreu, Weingart, \& Kwon, 2000; Komorita \& Parks, 1996; Van Lange, 1999). The predominant answer has been, in effect, that tough people are tough because they care more about winning, whereas soft people are soft because they just want to get along.

There is little doubt that values vary from person to person, and such variance explains meaningful differences in behavior. Yet, as learning theories dating back at least to Tolman (1932) have noted, and more recent social cognitive models of behavior (e.g., Bandura, 1986; Mischel \& Shoda, 1995) have recognized, people's behavior also reflects their expectations about the outcomes of their acts. People base their action not only on what they want but also on what they expect their behavior will achieve. The present article draws on this long tradition of work to pursue an expectancy-based account of assertiveness in conflict and negoti-

I thank Emily Amanatullah, Emily Bianchi, Jeremy Burrus, Adam Galinsky, Lara Kammrath, Elke Weber, and, especially, Brian Lickel for thoughtful comments on the research and manuscript.

Correspondence concerning this article should be addressed to Daniel Ames, Columbia Business School, Columbia University, 707 Uris Hall, 3022 Broadway, New York, NY 10027. E-mail: da358@columbia.edu ation that complements a motivational account. I argue that a person's expectations about the consequences of assertiveness predict their behavior in conflicts above and beyond the effects of their values. In other words, sometimes tough people are tough because they expect they can be, and soft people are soft because they believe they must be. The results of several studies support this account and hold implications for studying, counseling, and teaching about the dynamics of conflict.

\section{Values and Conflict}

Over the last half century, scholars of conflict have repeatedly linked interpersonal conflict behavior to underlying valuesvariously identified as preferences, concerns, priorities, and orientations. One active tradition of work revolves around dual-concern theory (e.g., Carnevale \& Pruitt, 1992) and motivational orientations (e.g., Messick \& McClintock, 1968), which posit that people vary in their attitudes about their own and their conflict partners' outcomes. Combinations of these dimensions yield different orientations such as "proself" (maximizing the difference between self and other) and "prosocial" (maximizing joint outcomes). Considerable research has linked these social value orientations to assertive behaviors in social dilemmas (e.g., McClintock \& Liebrand, 1988; Van Lange, 1999) and in conflict and negotiation (e.g., De Dreu \& Van Lange, 1995; De Dreu et al., 2000; Olekalns \& Smith, 2003).

Elsewhere, work in the interpersonal circumplex tradition has linked differences in concerns with agency and communion to assertiveness in conflict and negotiation (e.g., Amanatullah, Morris, \& Curhan, in press; Locke \& Sadler, 2007). Scholars have also invoked individual differences in need to belong (e.g., De Cremer \& Leonardelli, 2003) and face-saving concerns (e.g., White, 
Tynan, Galinsky, \& Thompson, 2004) to explain conflict behavior. In short, ample evidence shows that what people care about affects their assertiveness. There is no reason to question the usefulness of individual differences in values in accounting for interpersonal assertiveness, but there are reasons to suspect they are not the whole story. People's ability to explain differences in assertiveness could be enhanced by incorporating another construct psychologists have long recognized in other domains (e.g., Bandura, 1977): outcome expectancies.

\section{Outcome Expectancies and Assertiveness}

Although expectancies take many forms - ranging from stereotypes to attachment models (see Roese \& Sherman, 2007, for a review) - the present account stresses expected reactions by another to one's own assertive behavior. "If I do $X$," an actor thinks to her- or himself, "then this other person will do, think, or feel $Y$." Building on social cognitive models of behavior (e.g., Bandura, 1986; Mischel \& Shoda, 1995), such outcome expectancies have been examined in several contemporary traditions of work. Research on relational schemas has shown that behavior, such as the amount of anger displayed to a partner, depends in part on anticipated partner response (Fehr, Baldwin, Collins, Patterson, \& Benditt, 1999; see also Baldwin \& Dandeneau, 2005, for a recent review). A related area of inquiry has examined rejection sensitivity, which revolves around "anxious expectations" of interpersonal rejection and the associated activation of defensive responses, which can have negative or even self-fulfilling effects (e.g., Downey \& Feldman, 1996).

These and other areas of research suggest that expectancies will influence assertive behavior in interpersonal conflicts. Yet, the research to date has largely focused, for good reasons, on expectancies revolving around specific others, such as romantic partners (e.g., Kammrath \& Dweck, 2006), or has been based on attributes such as race or sex (e.g., Amanatullah, 2008; Bowles, Babcock, \& Lai, 2007). As such, the existence and impact of general assertiveness expectancies remains largely unexplored by expectancy researchers as well as by scholars of negotiation and conflict. The studies presented here pursue this topic directly.

\section{The Nature of Assertiveness Expectancies}

In the present account, I attempt to explain differing individual levels of assertiveness in everyday interpersonal conflict behaviors, including dyadic negotiations. Assertiveness is taken here to mean a person's tendency to stand up and speak out for their own interests and concerns, such as voicing opinions, making offers and concessions, and attempting to coerce or intimidate others (cf. Ames \& Flynn, 2007). To predict such behaviors with assertiveness expectancies, the nature of these beliefs (e.g., what is the relevant expectation to assess?) must first be clarified.

On the basis of prior work showing that interpersonal assertiveness often has a curvilinear, inverted- $U$-shaped effect on interpersonal relations (Ames \& Flynn, 2007), I expect that the majority of people will have curvilinear expectancies, assuming that they can push up to a point, but no further without incurring damage to their outcomes and/or relationships. For instance, in a negotiation, people may feel that making a moderately assertive opening offering could be effective, but at some point of heightened assertiveness, an opening could backfire, undermining both results and relationships. Although people in general may show this form of expectancy, individuals vary in what point they think they can push up to. Some may be very optimistic, assuming they can display high levels of interpersonal assertiveness before incurring costs. Others may be much more pessimistic, assuming that even modest levels of assertiveness could spell trouble. If this characterization is correct, then it would invite a research approach that gauges what individuals expect would happen when they push hard or give in.

An important initial goal of the present work, then, was to establish the nature of assertiveness expectancies. I suspect a majority of people will show a curvilinear pattern, believing that increasing assertiveness brings benefits up to a point. Just as important, though, I expect parameters of these expectancies (in particular, the assumed optimal level of assertiveness) to vary from person to person and that this variance can be of use in predicting behavior. An exploratory concern in the present article is how to measure these individual differences in assertiveness expectancies practically and effectively.

\section{Assertiveness Expectancies and Behavior}

The present account offers a central prediction: People make idiosyncratic forecasts of the social and instrumental consequences of a given forceful or acquiescent behavior, and, as social cognitive theories of personality suggest (Bandura, 1977; Mischel, 1973), these outcome expectancies shape behavior and can help researchers to predict it. It is possible that assertiveness is predominantly the product of values and that expectancies are simply reflections of these values. That is, those who expect high assertiveness to be harmful could simply be more concerned with preserving relationships. On the contrary, I expect that whereas assertiveness expectancies might be related to values, an independent expectancy-behavior link will remain after controlling for values. Two people could have identical values but differ in assertiveness simply because one expects a behavior would damage a relationship and the other does not.

The present account does not imply that values are unimportant, only that values will not account for all of the effects of expectancies. It is also worth noting that expectancies and values may interact: The expectancies that matter most in predicting behavior may be the ones concerning highly valued outcomes. Such interactions are examined in the analyses presented here.

\section{Plan of Study}

This article proceeds in two cumulative stages. The first stage is partly exploratory, examining how assertiveness expectancies might best be characterized and measured. The second stage is confirmatory, basing its operationalization of expectancies on the results of the first stage and attempting to explain assertive behavior with individual expectancies. Studies 1 and 2 comprise the first stage, inviting participants to literally chart out their expectancies and to consider a series of responses to hypothetical conflicts. Studies 3 and 4 comprise the second stage, using expectancies to predict behavior and outcomes in a dyadic negotiation and in the workplace. 


\section{Study 1}

In Study 1, I examined the nature of assertiveness expectancies by asking participants to draw lines showing social and instrumental outcomes as functions of a wide spectrum of assertiveness. The resulting figures were categorized by shape and analyzed for properties such as perceived optimal assertiveness. Measures of expectancies were compared with social values and self-reported assertiveness. I expected that the majority of lines would show inverted $U$ curvilinear shapes and that individual differences in these expectancies (e.g., perceived optimal assertiveness) would predict self-reported assertiveness after controlling for values.

\section{Method}

Participants. To assess the generalizability of results, Study 1 drew on two populations: paid university research participants and master's of business administration (MBA) students. No differences were expected between the groups. A total of 167 participants completed Study 1, including 104 MBA students, who took part in a course exercise involving the materials from Study 1, and 63 members of an East Coast university paid research participation program, the vast majority of whom were undergraduate students. Mean age for the MBA sample was $29.0(S D=3.0)$; $72(69.2 \%)$ were men. Of the 93 MBAs who indicated ethnicity, 52 (55.9\%) identified themselves as Caucasian, 29 (31.1\%) identified themselves as Asian or Asian American, 3 (3.2\%) identified themselves as Latino or Hispanic, and $3(3.2 \%)$ identified themselves as African American. Mean age for the paid participants was 21.6 $(S D=4.2)$, and $38(60.3 \%)$ identified themselves as women. Of the 56 who identified their ethnicity, 22 (39.3\%) identified themselves as Caucasian, $19(33.9 \%)$ identified themselves as Asian or Asian American, 8 (14.3\%) identified themselves as African American, 6 (10.7\%) identified themselves as Latino or Hispanic, and 1 (1.8\%) identified him- or herself as indigenous/ Native American.

Materials. Participants recorded their expectancies by drawing lines or curves related to two domains: social outcomes (e.g., being liked and trusted by others) and instrumental outcomes (e.g., getting favorable settlements and deal terms). Recent work by Ames and Flynn (2007) suggests that actual outcomes in these domains sometimes diverge, such that increasing assertiveness can sometimes yield instrumental gains and social costs. However, it was not clear, a priori, whether expectancies in one or the other of these domains would be a superior predictor of assertive behavior. As such, both were measured, and an exploratory consideration throughout the present article was whether one construct or the other better predicted behavior.

Participants were presented with hard-copy survey materials featuring $x$ - and y-axes forming the bottom and left sides of a chart. The bottom of the chart was labeled Assertiveness and ranged from 0 to 100 in increments of 10 . For the social outcomes chart, the $y$-axis was labeled social outcomes (trusted/liked by partner, partner wants to interact again in future). Participants were told that in this case, "partner" referred to the other (opposing) party in a conflict or negotiation. Instructions read as follows:

How do you think a person's assertiveness is related to their social outcomes in a conflict or negotiation, such as a negotiation partner's liking and trust for them, and their partner's interest in interacting with them again in the future? In the blank graph below, draw a line that represents how you see this relationship: what is the effect of assertiveness on social outcomes? The line can be any shape or angle you want, straight or curved. Your line should span the whole width of the chart, from the 0th percentile in assertiveness to the 100th percentile.

For the instrumental outcomes chart, the $y$-axis was labeled Results (payoffs, deal terms, and getting one's way). Instructions read as follows:

\begin{abstract}
How do you think a person's assertiveness is related to their results in a conflict or negotiation, non-social outcomes including their payoffs, deal terms, and the extent to which they get their way? In the blank graph below, draw a line that represents how you see this relationship: what is the effect of assertiveness on results? The line can be any shape or angle you want, straight or curved. Your line should span the whole width of the chart, from the 0th percentile in assertiveness to the 100th percentile.
\end{abstract}

The order of charts was counterbalanced. Pilot work revealed that participants found the task meaningful and captured assumptions that they "knew" but otherwise found difficult to articulate. Pilot work using $z$ scores for the axes led to confusion; participants found the task most interpretable and meaningful when the axes were described in terms of percentiles for their peer group.

Along with the line drawing, participants indicated their own assertiveness as well as their own social values. For self-rated assertiveness, participants completed a percentile measure:

Compared to your classmates, what percentile do you think you're in for assertiveness? 50\% would mean average assertiveness compared to classmates, while $10 \%$ would mean you're in the lowest $10 \%$, and $90 \%$ would mean you're in the top $10 \%$ in terms of assertiveness.

For social outcome values, participants were asked, "Sometimes, people care more about their results or more about their social outcomes. How would you describe your general balance of these preferences? Do you generally care about results or outcomes more-or care about them the same?" Participants rated their response on a 13-point scale ranging from 1 (Much more about results) to 13 (Much more about social outcomes), with the midpoint, 7, labeled About the same. The order of the self-reported assertiveness and value questions was counterbalanced, as was their placement before versus after the line drawing exercise.

\section{Results}

Results for the two participant populations were very similar. As such, the samples were collapsed for analyses reported below except where noted.

Characterizing expectancies. Study 1 was concerned with characterizing assertiveness expectancies with an eye toward identifying effective ways to measure them. As anticipated, many participants expected curvilinear effects of assertiveness on both social and instrumental outcomes such that both extremely low and high levels of assertiveness were associated with lower outcomes. The majority of both paid and MBA participants drew inverted$U$-shaped functions for social and instrumental outcomes (see Table 1). The mean anticipated outcomes across all participants are shown in Figure 1. It is worth noting that although most participants indicated an inverted $U$ curve, a sizable minority (33\%) drew 
Table 1

Frequency of Shapes of Individual Curves Depicting Outcomes as a Result of Assertiveness In Study 1

\begin{tabular}{|c|c|c|c|c|c|c|}
\hline \multirow[b]{2}{*}{ Variable } & \multicolumn{3}{|c|}{ Social outcomes } & \multicolumn{3}{|c|}{ Instrumental outcomes } \\
\hline & $\begin{array}{c}\text { Paid } \\
\text { participants }\end{array}$ & $\begin{array}{c}\text { MBA } \\
\text { participants }\end{array}$ & $\begin{array}{c}\text { All } \\
\text { participants }\end{array}$ & $\begin{array}{c}\text { Paid } \\
\text { participants }\end{array}$ & $\begin{array}{c}\text { MBA } \\
\text { participants }\end{array}$ & $\begin{array}{c}\text { All } \\
\text { participants }\end{array}$ \\
\hline Inverted U & 77.8 & 63.5 & 68.9 & 54.0 & 66.3 & 61.7 \\
\hline Downward convex & 1.6 & 2.9 & 2.4 & 0.0 & 1.0 & 0.6 \\
\hline Downward concave & 1.6 & 10.6 & 7.2 & 0.0 & 1.0 & 0.6 \\
\hline Downward straight & 3.2 & 5.8 & 4.8 & 1.6 & 1.0 & 1.2 \\
\hline Upward convex & 7.9 & 7.7 & 7.8 & 20.6 & 12.5 & 15.6 \\
\hline Upward concave & 3.2 & 3.8 & 3.6 & 7.9 & 6.7 & 7.2 \\
\hline Upward straight & 3.2 & 2.9 & 3.0 & 15.9 & 6.7 & 10.2 \\
\hline Other & 1.6 & 1.9 & 1.8 & 0.0 & 4.8 & 3.0 \\
\hline
\end{tabular}

Note. Cells represent percentage of participants (paid participant $n=63$, MBA participant $n=104$ ). Inverted $\mathrm{U}$ functions showed clear downturns at both the low and high ends; convex lines showed an upward bulge; concave lines showed a downward bulge; straight lines showed no curvature. MBA = Master's of business administration.

upward lines with no discernable downturn for instrumental outcomes as a function of assertiveness (see Table 1).

The prevalence of curvilinear expectancies suggests that one way to capture individual differences in assertiveness expectancies is to gauge individuals' perceived optimal level of assertiveness, the point of assertiveness that a person believes maximizes outcomes (i.e., pushing up to this point brings net benefits, but pushing beyond it undermines results). For a linear upward slope for instrumental outcomes, this "instrumental optimum" was 100. For an inverted $\mathrm{U}$ line in which social outcomes were greatest at an assertiveness level of 60, this "social optimum" was 60 . Higher optima thus indicated expectancies that higher levels of assertiveness would afford better outcomes. Put another way, perceived optimal assertiveness reflects the point at which people believe they can gainfully push up to. Analyses reported below test whether these optimum measures predicted self-reported assertive-

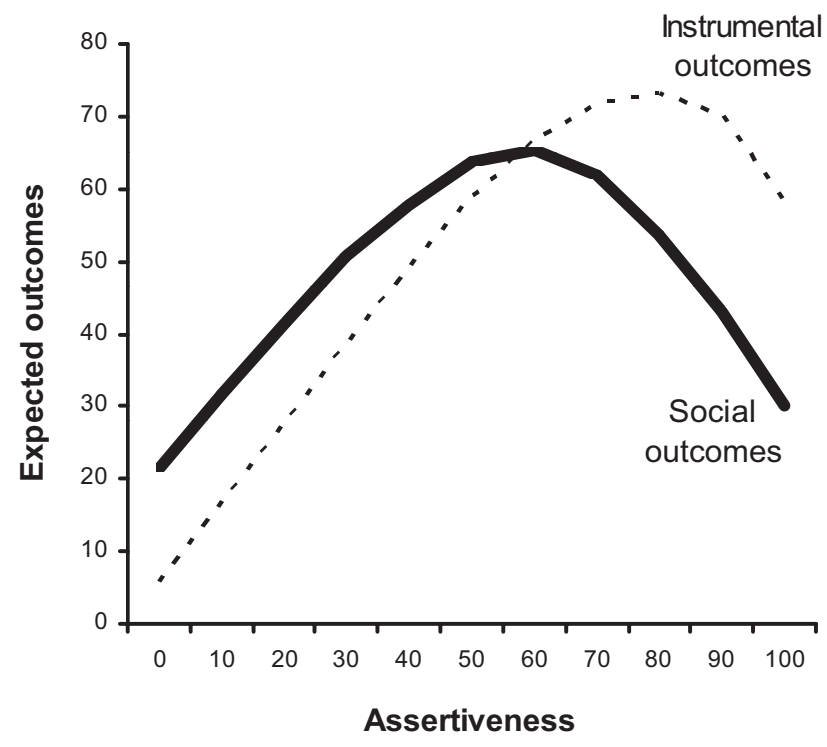

Figure 1. Mean ratings for expected social and instrumental outcomes across levels of assertiveness in Study 1. ness, but before attempting to predict assertiveness, additional explorations of assertiveness expectancies as a construct and measure are worth considering.

Individual differences in expectancies are necessary to explain individual differences in behavior. Thus, a natural question is: Are there areas in which the variance in expectancies is more or less pronounced? To gauge this, I plotted the mean level of anticipated social and instrumental outcomes across the spectrum of assertiveness, as well as values one standard deviation above and below the mean, in the top half of Figure 2. As can be seen in the top left portion of the figure, social expectancies seem to have somewhat greater variance at the extremes of assertiveness. In the top right portion of the figure, the variance of instrumental expectancies seems clearly higher at the very highest levels of assertiveness. In other words, people appeared to agree that low assertiveness leads to poor instrumental outcomes. However, people diverged about the instrumental rewards they anticipated for the highest levels of assertiveness.

Expectancy constructs. On the basis of the foregoing analyses attempting to characterize assertiveness expectancies, three types of measures were computed as independent variables that might predict self-reported assertiveness. The first of these was perceived optimal assertiveness, as described above (i.e., the perceived level of assertiveness that maximized social or instrumental outcomes). The other two measures of expectancies were based on expected outcomes for extreme low and high levels of assertiveness. Specifically, anticipated outcomes (as reflected in participants' line drawings) for the 0 and 10 levels of assertiveness were averaged to compute measures of expectancies for low assertiveness. Likewise, anticipated outcomes for the 90 and 100 levels of assertiveness were averaged to compute measures of expectancies for high assertiveness. ${ }^{1}$ Separate measures were computed for social and instrumental outcomes.

Predicting assertiveness. The constructs described above were analyzed for their ability to predict self-reported assertiveness. This affords a test of the prediction that expectancies predict

\footnotetext{
${ }^{1}$ Results were very similar for just the extreme values, 0 and 100 , as well as an average of three endpoints $(0,10$, and 20;80, 90, and 100).
} 


\section{Where do expectancies vary most from person to person?}

Plots of means and one standard deviation above/below mean

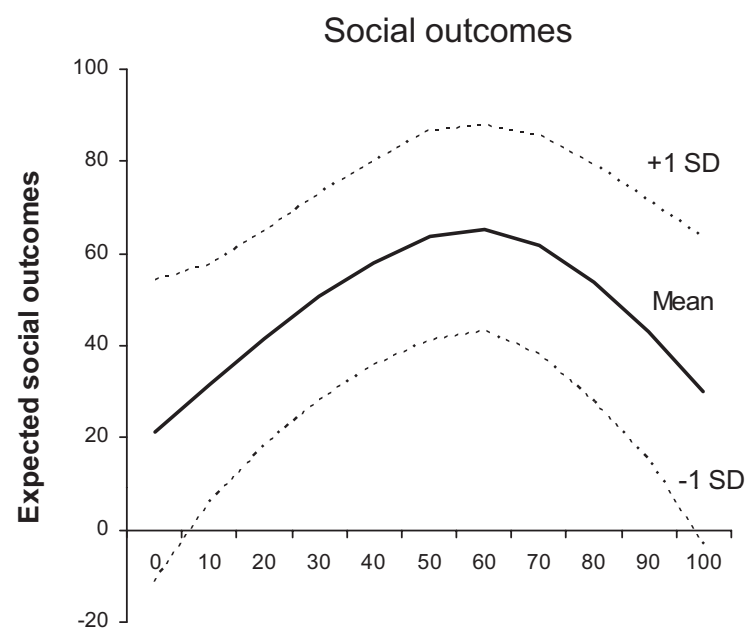

Assertiveness

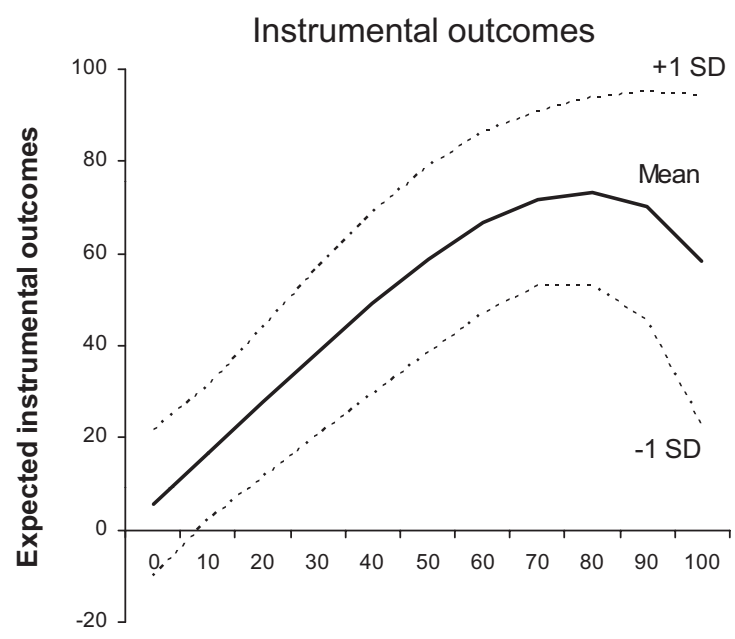

Assertiveness

\section{Which expectancies best predict self-reported assertiveness?}

Across participant correlation between self-reported assertiveness and anticipated outcomes for each level of assertiveness

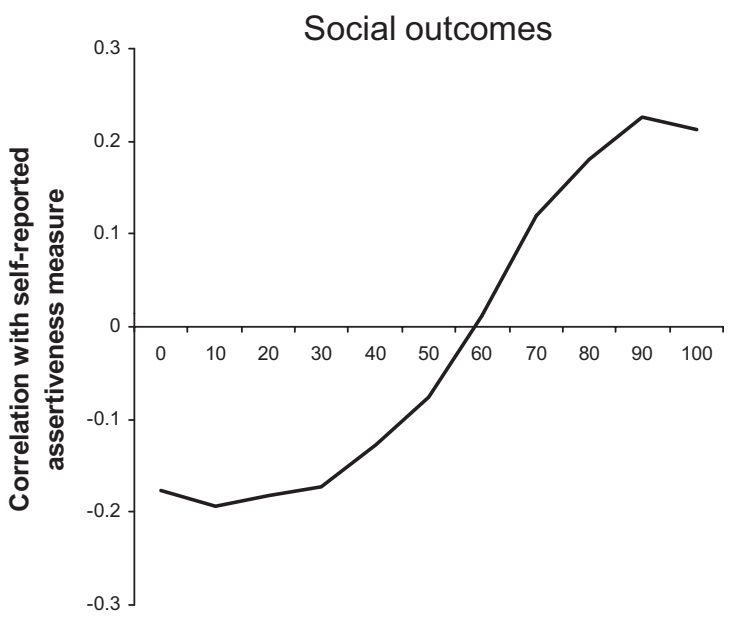

Assertiveness

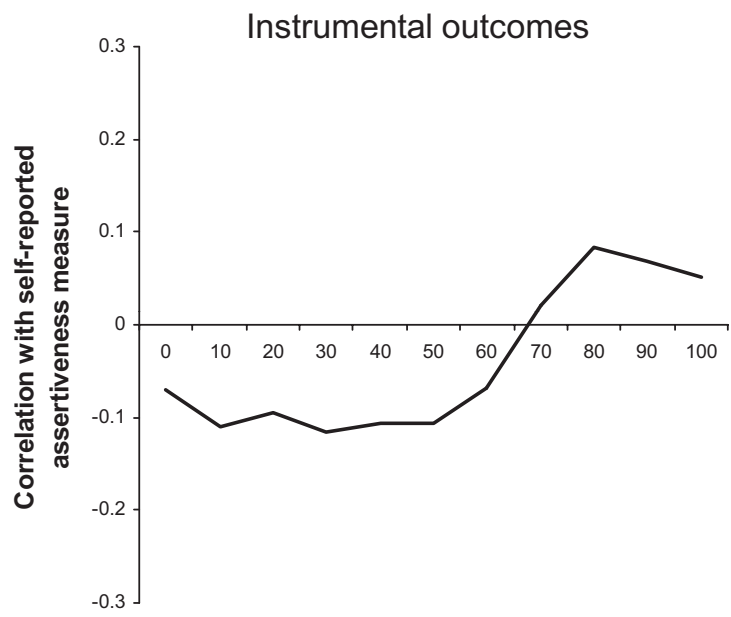

Assertiveness

Figure 2. Variance in expectancies and correlation with optimum measures in Study 1.

assertiveness and also informs the exploratory effort to identify what measures of expectancies are the most effective predictors.

As shown in Table 2, the social optimum measure (perceived optimal assertiveness for social outcomes) was positively corre- lated with self-rated assertiveness, meaning that individuals who expected they could push harder before incurring social costs were also more likely to self-report higher levels of assertiveness. In addition to social optimum, both low- and high-assertiveness social 
Table 2

Correlations Between Curve-Based Constructs, Values, and Self-Rated Assertiveness in Study 1

\begin{tabular}{|c|c|c|c|c|c|c|c|c|}
\hline Variable & 1 & 2 & 3 & 4 & 5 & 6 & 7 & 8 \\
\hline 1. Social optimum & - & & & & & & & \\
\hline $\begin{array}{l}\text { 2. Social expectancies for } \\
\text { low assertiveness }\end{array}$ & $-.80^{\text {*** }}$ & - & & & & & & \\
\hline 3. Social expectancies for & & & & & & & & \\
\hline high assertiveness & $.68^{* *}$ & $-.42^{* *}$ & - & & & & & \\
\hline 4. Instrumental optimum & $.22^{* * *}$ & $-.15^{*}$ & $.24^{* * *}$ & - & & & & \\
\hline 5. Instrumental expectancies & & & & & & & & \\
\hline $\begin{array}{l}\text { for low assertiveness } \\
\text { 6. Instrumental expectancies }\end{array}$ & -.12 & $.26^{* *}$ & -.02 & $-.48^{* *}$ & - & & & \\
\hline $\begin{array}{l}\text { 6. Instrumental expectancies } \\
\text { for high assertiveness }\end{array}$ & $.19^{*}$ & -.08 & $.40^{* * *}$ & $.65^{* * *}$ & $-.16^{*}$ & - & & \\
\hline 7. Social outcome values & -.03 & -.01 & -.01 & -.04 & .00 & -.03 & - & \\
\hline 8. Self-rated assertiveness & $.25^{* * *}$ & $-.19^{*}$ & $.23^{* *}$ & $.14^{\dagger}$ & -.09 & .06 & $-.25^{* *}$ & - \\
\hline$M$ & 54.12 & 26.62 & 36.65 & 78.74 & 11.27 & 64.27 & 6.15 & 60.71 \\
\hline$S D$ & 29.79 & 28.40 & 29.47 & 21.59 & 14.44 & 28.88 & 2.42 & 18.11 \\
\hline
\end{tabular}

Note. $n=162$.

${ }^{\dagger} p<.10 . \quad{ }^{*} p<.05 . \quad{ }^{* *} p<.01$.

expectancies were predictive. Those who believed very low levels of assertiveness had more positive social outcomes reported lower levels of personal assertiveness. Those who believed very high levels of assertiveness had more positive social outcomes reported higher levels of personal assertiveness. It is worth noting that when both low- and high-assertiveness social expectancies were entered as independent variables in a regression predicting self-reported assertiveness, high assertiveness expectancies remained significantly predictive $(\beta=.18), t(163)=2.15, p<.05$, but low assertiveness expectancies appeared to lose their predictive power $(\beta=-.11), t(163)=1.36, p=.18$. In short, several operationalizations of social expectancies were predictive of self-reported assertiveness (see the bottom half of Figure 2 for additional detail on how expectancies at different levels of assertiveness predicted self-reported assertiveness). As shown in Table 2, the instrumental expectancy measures were not significantly correlated with selfreported assertiveness.

Independent effect prediction. With the social outcome value measure, Study 1 also afforded a test of whether the expectancyassertiveness link remained after controlling for values. As might be expected, the value measure (relative concern with social outcomes) was negatively correlated with self-rated assertiveness, though this measure was not significantly correlated with the expectancy constructs (see Table 2). To test the independent effect prediction (that the predictive power of expectancies would remain after controlling for values), regression analyses were conducted, predicting self-reported assertiveness with social expectancies and values simultaneously. In a first model, both social optimum $(\beta=$ $.25), t(160)=3.41, p<.01$, and values $(\beta=-.22), t(160)=$ $-2.98, p<.01$, were predictive. In a second model, both social expectancies for low assertiveness $(\beta=-.20), t(160)=-2.68$, $p<.01$, and values $(\beta=-.24), t(160)=-3.22, p<.01$, were predictive. In a third model, both social expectancies for high assertiveness $(\beta=.23), t(160)=3.11, p<.01$, and values $(\beta=$ $-.24), t(160)=-3.19, p<.01$, were predictive. These results are consistent with the independent effect prediction.

Additional models were run to test for the possible interaction between expectancies and values (i.e., that social outcome values affect whether social or instrumental expectancies will be more predictive of self-rated assertiveness). A wide range of models revealed no significant interaction effects.

\section{Discussion}

A central goal of Study 1 was to characterize peoples' expectancies about interpersonal assertiveness, attempting to shed light on how they might be conceptualized and measured effectively in the service of predicting individual differences in assertive behavior. As anticipated, and echoing what Ames and Flynn (2007) found in their work on the actual impact of assertiveness, the majority of participants in Study 1 displayed curvilinear social and instrumental expectancies, assuming they could push up to a point, beyond which costs would begin to outweigh benefits. More important, these expectancies varied from person to person. One point of variance was the perceived optimum, the point to which people believed they could push - that is, the level of assertiveness that maximized outcomes. Analyses also revealed that people tended to agree more on the expected outcomes for moderate assertiveness, diverging more significantly in their forecasts of what would follow from extreme low or high assertiveness.

Study 1 also afforded an initial test of whether expectancies predict assertiveness. All three measures of social expectancies predicted self-reported assertiveness, including social optimum (perceived optimal assertiveness for social outcomes), social expectancies for low assertiveness, and social expectancies for high assertiveness. These links remained after controlling for social outcome values (i.e., concern with relationships vs. instrumental outcomes). Indeed, social expectancies were not significantly correlated with outcome values, casting doubt on the notion that the two constructs simply reflect the same underlying attitude.

Instrumental expectancies did not predict self-rated assertiveness in Study 1. It could be that instrumental expectancies simply matter less to people than social ones. People may prioritize the preservation of relationships and find perceived relational costs relatively abhorrent (e.g., Baumeister \& Leary, 1995). It could also be, though, that the approach taken in Study 1's line drawing 
paradigm was vague in terms of what was meant by "results." Perhaps when perceivers consider a concrete situation with specific instrumental outcomes that they can identify (e.g., a starting salary in a job negotiation, as in Study 2), the ability of instrumental expectancies to predict behavior will be greater.

\section{Study 2}

Study 1 shed light on how assertiveness expectancies could be operationalized, and links between expectancies and self-reported assertiveness were found. Although encouraging, the measures used were rather decontextualized: The independent measures of expectancies were based on line drawings about the general impact of assertiveness, and the dependent measure was a self-report of general assertiveness. Before committing to an operationalization of expectancies on the basis of line drawings alone, Study 2 was used to examine expectancies with greater specificity and contextual detail. Study 2's more concrete approach measured expectancies and behavioral preferences in a series of specific scenarios, such as a salary negotiation.

Study 2 had two goals. A first goal was to test the prediction that expectancies would predict assertiveness and that this link will remain after controlling for relevant values; accordingly, additional measures of relevant values were introduced. The second goal was to extend the exploratory work from Study 1 on gauging assertiveness expectancies. Recall that in Study 1, expected outcomes for low and high levels of assertiveness showed considerable variance and were variously linked to self-reported behavior. Accordingly, the focus in Study 2 was on expected outcomes for low and high levels of assertiveness as a way of gauging expectancies. In Study 2, I used expectancies in particular contexts to predict behavioral preferences in those same contexts. Both social and instrumental expectancies were measured, allowing an assessment of whether either or both were predictive.

\section{Method}

Participants. Sixty-seven working managers enrolled in a business education program in the United States participated in Study 2 as part of a session exercise. Mean age was $37.2(S D=$ 5.27), and $54(80.6 \%)$ identified themselves as male. Forty-seven (70.1\%) identified themselves as Caucasian, 15 (22.4\%) identified themselves as Asian or Asian American, 1 (1.4\%) identified himor herself as African American, 1 (1.4\%) identified him- or herself as Native American, and $3(4.5 \%)$ did not identify their ethnicity.

Assertiveness scenarios. Scenarios were used to assess both assertiveness expectancies (the independent measure) and behavioral preferences (the dependent measure). Participants responded to three scenarios presented in counterbalanced order: one featuring a salary negotiation, one regarding asking a freelancer for help under a deadline, and one concerning disagreeing with a colleague in a team meeting. Each scenario featured a beginning ("Imagine that you were negotiating with a potential employer...") and then three endings featuring low, medium, and high assertive behaviors. The scenarios and endings are presented in the Appendix. Endings were presented in counterbalanced order.

After each ending, participants rated expected social outcomes ("How much do you think the [manager, freelancer, work colleague] would like, trust, and want to interact with you in the future?") on an 11-point scale ranging from 1 (strong dislike, distrust, disinterest) to 11 (strong like, trust, and interest). Participants also rated expected instrumental outcomes (e.g., "How well do you think you would do in terms of final negotiated salary") on an 11-point scale ranging from 1 (extremely poorly) to 11 (extremely well). These anticipated social and instrumental outcomes at the scenario level were taken as the independent variable measures of expectancies. That is, expectancies for high assertiveness were the ratings for social outcomes and instrumental outcomes for the high assertiveness ending. As noted in the Results section, separate multilevel models were run using low, medium, and high assertiveness expectancies as predictors, as well as all of these expectancies as simultaneous predictors.

After rating all three endings for a given scenario, participants were asked to consider the endings and "rank these three responses for how likely you would be to use them." Participants were told to give a 1 for the response they would be most likely to use and a 3 for the response they would be least likely to use. These rankings were used to construct a dependent measure of preferred assertiveness at the scenario level. This was computed as the level of assertiveness (low, moderate, or high) that a respondent most preferred for a given scenario. Low assertiveness was assigned a value of 1 , moderate assertiveness a value of 2 , and high assertiveness a value of 3 . Thus, participants' preferences for higher assertive responses in the scenarios were reflected in higher scores for preferred assertiveness. The average preferred assertiveness across the scenarios (including 198 valid responses; three per participant) was $2.36(S D=0.50)$. In the majority of cases $(61.6 \%)$, respondents indicated a preference for the moderate assertiveness response. In nearly all of the remaining cases (37.4\%), respondents indicated a preference for the high assertiveness response; respondents most preferred the low assertiveness response in only $1 \%$ of cases.

Value measures. In a separate exercise completed earlier in the business education program, participants responded to items gauging relevant values. Two items gauged social outcome values, a comparative concern for relational outcomes over instrumental ones, including "I care more about getting along with others than 'winning' each conflict or disagreement" and "In a conflict, I generally care more about results (getting my way) than social outcomes (my partner's liking and trust for me, and interest in interacting with me again in the future)." Participants rated these items on 5-point scales ranging from 1 (disagree strongly) to 5 (agree strongly).

In addition, participants completed a measure of unmitigated communion, a construct involving extreme concern for others (Fritz \& Helgeson, 1998) that has been linked to negotiation outcomes (Amanatullah et al., in press). Because of time constraints, five items were sampled from the original nine-item scale ("I always place the needs of others above my own," "For me to be happy, I need others to be happy," "I can't say no when someone asks me for help," "Even when exhausted, I will always help other people," "I often worry about others' problems"). Items were rated on 5-point scales ranging from 1 (disagree strongly) to 5 (agree strongly).

Finally, in a separate exercise, participants indicated their social value orientations (SVO), a commonly used tool for measuring values relevant to conflict and negotiation (e.g., Van Lange, 1999). The SVO measure gathered responses to a series of nine decomposed 
games (Messick \& McClintock, 1968; see also Van Lange, Otten, De Bruin, \& Joireman, 1997). In each game, participants chose between three alternative pairs of outcomes, each representing one of the three SVOs: prosocial (maximizing joint gain), individualist (maximizing own gain without regard for the other's outcome), and competitive (maximizing the difference between own and other outcome). The number of responses consistent with each orientation was used to create continuous measures for each of the three orientations.

\section{Results}

The primary objective in Study 2 was to use expectancy and other measures to predict preferred assertiveness. Due to the nested nature of the data, multilevel modeling was used. It was not expected that scenario would matter to the expectancyassertiveness link, yet controlling for scenario could clarify the results. A Level 1 model was created at the scenario level (three per participant), featuring preferred assertiveness as the dependent measure (i.e., the level of assertiveness ranked as most preferred, where low assertiveness $=1$, moderate assertiveness $=2$, and high assertiveness $=3$ ). This was predicted by dummy codes for scenario (two dummy codes to reflect the three scenarios) and social and instrumental expectancies for the given scenario. Separate models featuring low, moderate, and high assertiveness expectancies were run, as described in the paragraph below. The Level 2 model at the person level featured effects for the value measures, including unmitigated communion, SVO competitive, and SVO prosocial (given the fixed sum structure of the SVO measure, the SVO individualist variable was withheld from the model because it is equivalent to the residual of the SVO competitive and prosocial measures). In addition, because the social value items (getting along with others, caring about results) were only modestly correlated with one another, $r(66)=.45$, they were both entered as independent predictors in the Level 2 model.

Using hierarchical linear modeling (HLM) 6 to compute least squares estimates of fixed effects (Raudenbush, Bryk, Cheong, \& Congdon, 2004), a first model featured high-assertiveness social and instrumental expectancies in Level 1 (the scenario level). In this model, both social and instrumental expectancies were significant predictors (unstandardized coefficents of $0.047, t[189]=$ $3.52, p<.01$; and, 0.058, $t[189]=4.78, p<.01$, respectively) as was SVO competitive (0.046), $t(189)=4.79, p<.01)$. Scenarios did not show a significant effect, nor did the other value measures. A second model featured low-assertiveness social and instrumental expectancies in Level 1. In this model, instrumental expectancies were not a significant predictor, but social expectancies were $(-0.026), t(189)=2.03, p<.05$, as was SVO competitive $(0.054), t(189)=3.94, p<.01$. None of the other value measures were significantly predictive. A third model featured moderate assertiveness social and instrumental expectancies in Level 1. In this model, social expectancies were not a significant predictor, but instrumental expectancies were $(-0.047), t(189)=2.81, p<.01$, as was SVO competitive (0.046), $t(189)=5.94, p<.01$. All six measures of expectancies (in Level 1) were used in a final model as well as the individual difference measures (in Level 2). SVO competitive was predictive $(0.046), t(184)=5.14, p<.01$, as were low-assertiveness social expectancies $(-0.023), t(184)=$ 2.21, $p<.05$; medium assertiveness instrumental expectancies
$(-0.042), t(184)=3.12, p<.01$; high assertiveness social expectancies $(0.040), t(184)=2.89, p<.01$; and high assertiveness instrumental expectancies $(0.064), t(184)=5.77, p<.01$.

In summary, across these models, both social and instrumental expectancies for high assertiveness and SVO competitive were strongly predictive of preferred assertiveness. The multilevel analyses also showed significant effects for low-assertiveness social expectancies and moderate assertiveness instrumental expectancies. To explore the possible interaction of values and expectancies, additional multilevel models were run to test for interactions between the expectancy measures and the social value measures (care about results, important to get along) as well as unmitigated communion. No significant interactions emerged.

A final exploratory analysis was conducted, starting with the dependent variable (averaging preferred assertiveness across the three scenarios) and identifying individuals in the lowest and highest thirds of assertiveness. Contrasting the expectancies for these two groups showed similar social and instrumental expectancies for low and medium assertiveness responses. However, the groups differed markedly in their expectancies for high assertiveness behaviors where highly assertive individuals expected significantly more positive outcomes.

\section{Discussion}

Study 2 built on Study 1 by having participants review a series of concrete scenarios involving interpersonal conflict. Participants indicated their preferred level of assertiveness in response to each scenario (ranging from low to high assertiveness) and also recorded their expected social and instrumental outcomes. As anticipated, expectancies predicted preferred assertiveness: Those who were comparatively optimistic about outcomes for high assertive behaviors were more likely to prefer highly assertive responses. Whereas an effect only for social expectancies was found in Study 1, effects for both social and instrumental expectancies in predicting assertiveness were shown in Study 2. These expectancyassertiveness links remained after controlling for value measures, including SVO competitive, which also predicted behavioral preferences. In short, preferences for responding in an assertive way were predicted not only by interpersonal motivations but also by an individual's expectations for how costly or beneficial a highly assertive response would be.

Moderate and low assertiveness expectancies were not consistently or strongly predictive of behavioral preferences. The overall pattern of results, and exploratory analyses of expectancies, reinforced what Study 1 suggested: Expected outcomes for high assertive behaviors seem to be a promising operationalization of idiosyncratic assertiveness expectancies.

\section{Measuring Expectancies to Predict Assertiveness}

Studies 1 and 2 provided initial support for the predicted link between expectancies and assertiveness. Another motivation behind these studies was to explore ways in which assertiveness expectancies might be meaningfully and efficiently measured. The results warrant brief consideration before continuing on to additional tests of the expectancy-assertiveness link.

An advantage of the line drawing paradigm in Study 1 is that it yielded a complete line or curve that identified a perceived optimal 
level of assertiveness. Conceptually, this is a compelling way of reflecting expectancies, yet it is decontextualized in its representation of both behaviors and outcomes. Using more concrete behaviors and outcomes has appeal but presents a challenge in that detailing a full and nuanced range of increments in assertiveness (e.g., 10 possible detailed responses in an interpersonal conflict) could make for a cumbersome measure.

Expected outcomes for highly assertive behaviors seem like a promising measure of expectancies. In Study 1, person-to-person variance appeared to be highest in anticipated outcomes for extreme behaviors, especially highly assertive behaviors (see top half of Figure 2). In terms of predicting self-reported assertiveness, expected social outcomes for highly assertive behaviors fared best (see the bottom of Figure 2). In Study 2, with concrete scenarios, expected social and instrumental outcomes for high assertiveness were consistently predictive of behavioral preferences, whereas expected outcomes for low and moderate assertiveness were generally not. I return in this article's General Discussion section to the matter of why expectancies for highly assertive behavior might have comparatively robust predictive power.

In Studies 3 and 4, I attempted to link high assertiveness expectancies to real-world assertive behavior. I used concrete scenarios in both studies like those in Study 2 to gauge expectancies, using these expectancies as an independent measure to predict partner reports of participant assertiveness in a dyadic negotiation (Study 3) and coworker reports of participant assertiveness in the workplace (Study 4). Thus, whereas expectancies in particular contexts were used to predict behavior in those fictional contexts in Study 2, expectancies in one set of fictional contexts were used to predict behaviors in somewhat different real-world contexts in Studies 3 and 4.

\section{Study 3}

Building on Studies 1 and 2, Study 3 operationalized expectancies as anticipated social and instrumental outcomes for high assertiveness behaviors in conflict scenarios (measured in domains different from that featured in the negotiation and collected several weeks in advance). It was expected that these expectancies would predict negotiation behavior and outcomes, controlling for the effect of values. In addition, Study 3 went beyond Study 2 by including additional value and dispositional measures related to assertiveness, including conflict styles and relevant personality facets.

\section{Method}

Participants. Sixty-four MBA students, with a mean age of $29.1(S D=2.7)$, took part in Study 3 during an elective course on negotiation. Twenty-nine participants $(45 . \%)$ were women. Fortyone participants $(64.1 \%)$ identified themselves as Caucasian, 15 $(23.4 \%)$ identified themselves as Asian or Asian American, 3 $(4.7 \%)$ identified themselves as Latino or Hispanic, and 1 (1.6\%) identified him- or herself as African American.

Expectancy measures. Prior to the beginning of the course, participants indicated their expectancies for two scenarios (two of the three featured in Study 2). One featured responses to a belowmarket-rate salary offer; the other featured responses to managing a freelance employee during a severe project deadline. The scenarios each featured a common beginning and three behavioral conclusions varying in assertiveness, as shown in the Appendix.
For each behavior (low, medium, and high assertiveness), participants indicated expected social outcomes on an 11-point scale ranging from 1 (strong dislike, distrust) to 11 (strong like, trust). For the salary negotiation, the item read, "How much do you think the manager would like, trust, and want to interact with you in the future?" For the freelancer scenario, the item read, "How much do you think the freelancer would like, trust, and want to interact with you in the future?"

Participants also indicated expected instrumental outcomes on an 11-point scale ranging from 1 (extremely poorly) to 11 (extremely well). For the salary negotiation, the item read, "How well do you think you would do for yourself in terms of final negotiated salary?" For the freelancer scenario, the item read, "How well do you think you would do for yourself in terms of project outcomes (completed profiles, budget, time, etc.)?"

Conflict measures. A week after the expectancy measure, participants completed a variety of other individual-difference measures. Two of these were focused on the domain of conflict: SVO (as measured in Study 2) and the Thomas-Kilmann Conflict Mode Instrument, or TKI. The TKI gauges a person's style for resolving conflict along five orientations: accommodating, avoiding, collaborating, competing, and compromising (Thomas \& Kilmann, 2002). These orientations are located by crossing two dimensions, similar to those in the SVO: an individual's emphasis on satisfying her or his own concerns, and an individual's emphasis on satisfying the concerns of the other party. The TKI contains 30 paired statements that describe two of the five conflict orientation modes. For each pair, respondents were asked to select the statement that more aptly described them. For instance, one pair asks participants to choose between "I propose a middle ground" (Compromising) and "I press to get my points made" (Competing). Possible scores on each of the five conflict orientations ranged from 0 to 12 .

Other individual-difference measures. Along with the conflict measures, participants completed several other value and personality measures that might account for assertive behavior. These included a number of eight-item subscales of the Revised NEO Personality Inventory (Costa \& McCrae, 1992), rated on 5-point scales ranging from 1 (disagree strongly) to 5 (agree strongly). Several relevant facets of Extraversion were measured, including Assertiveness (e.g., "I am dominant, forceful, assertive") and Warmth (e.g., "I'm known as a warm and friendly person"). Several relevant facets of Agreeableness were also measured, including Trust (e.g., "My first reaction is to trust people"), Compliance (e.g., "I hesitate to express my anger even when it's justified"), and Altruism (e.g., "I go out of my way to help others if I can"). In addition, Angry Hostility, a facet of Neuroticism, was measured (e.g., "I often get angry at the way people treat me"). In addition, participants completed measures of unmitigated communion (five items) and social outcome values (two items), as in Study 2.

Negotiation measures. Two weeks after the expectancy assessment, participants were randomly paired and completed a two-person face-to-face role play negotiation. One participant in each pair played the role of a company selling a factory; the other participant played the role of a buyer. Both participants received the same general information about the case, including the points that the seller purchased the plant for $\$ 15$ million several years ago, that a recent appraisal of the factory indicated a value of $\$ 19$ million, and that a similar but new plant recently sold for \$26 million. The buyer received confidential information indicating 
that their best alternative to a negotiated agreement with their partner (also known as a BATNA) was $\$ 25$ million for building a new plant. The seller received confidential information indicating that their BATNA was $\$ 17$ million for stripping and scrapping the plant. Thus, the buyer should not have paid more than $\$ 25$ million, and the seller should not have accepted less than $\$ 17$ million. No other issues were introduced or allowed, making this a single-issue distributive (i.e., zero-sum) negotiation focused on sale price. All pairs reached a settlement.

After the negotiation (usually lasting 20-30 min) was finished, participants completed ratings of their partner. An initial item gauged perceived assertiveness ("How assertive was your partner during the negotiation?") on a 12-point scale ranging from 1 (not assertive at all) to 12 (extremely assertive). Next, participants rated whether they saw their partner as under- versus overassertive on a 5-point scale, including 1 (very underassertive), 2 (somewhat underassertive), 3 (appropriately assertive), 4 (somewhat overassertive), and 5 (very overassertive).

Participants subsequently rated their partner's behaviors on a 6-point scale ranging from 1 (not at all/never) to 6 (a great deal/always). Items included "They made extreme or bold offers," "They made meaningful concessions," "They displayed competitive, aggressive behavior," "They took steps to avoid and/or diffuse any tension," and "They revealed helpful or important information." Finally, participants rated their trust for their partner ("Do you feel like you could trust your partner in future negotiations?") and their attitudes about future interaction with their partner ("Do you look forward to future interactions and teamwork with your partner?") on 12-point scales ranging from 1 (strongly disagree) to 12 (strongly agree).

\section{Results}

Means and reliabilities for the measures are shown in Table 3. The two social outcome value items were only modestly correlated, $r(64)=.31, p=.01$, and are thus analyzed separately.

Table 3

Scale Means and Reliabilities in Study 3

\begin{tabular}{lcccc}
\hline \multicolumn{1}{c}{ Construct } & Items & $M$ & $S D$ & $\alpha$ \\
\hline SVO Prosocial & - & 3.41 & 3.81 & - \\
SVO Individualist & - & 4.72 & 3.95 & - \\
SVO Competitive & - & 0.87 & 2.50 & - \\
TKI Collaborate & - & 5.25 & 2.02 & - \\
TKI Compete & - & 5.36 & 3.22 & - \\
TKI Accommodate & - & 5.86 & 2.30 & - \\
TKI Avoid & - & 6.03 & 3.00 & - \\
TKI Compromise & - & 7.42 & 2.17 & -64 \\
Unmitigated communion & 5 & 3.32 & 0.64 & .64 \\
NEO Assertiveness & 8 & 3.29 & 0.74 & .81 \\
NEO Warmth & 8 & 4.05 & 0.66 & .81 \\
NEO Angry Hostility & 8 & 2.70 & 0.87 & .84 \\
NEO Trust & 8 & 3.46 & 0.84 & .89 \\
NEO Compliance & 8 & 3.05 & 0.57 & .60 \\
NEO Altruism & 8 & 3.99 & 0.52 & .70 \\
\hline
\end{tabular}

Note. Number of items and alpha values are shown only for Likert-rating scales. SVO = Social Value Orientation; TKI = Thomas-Kilmann Conflict Mode Instrument; dashes represent scales where the number of items is not necessarily meaningful.
Expectancies. The independent variable of expectancies was based on responses to the conflict scenarios. Across a wide range of results and consistent with Study 2, expected outcomes for the high assertiveness behaviors (averaged across the two scenarios) were substantially and consistently more predictive than those for the low or medium assertiveness behaviors. ${ }^{2}$ As such, the results focus on these measures as the operationalization of expectancies. For social outcomes at high assertiveness, mean expectancies on the 11-point scale were $5.20(S D=1.66)$; for instrumental outcomes at high assertiveness, mean expectancies were $7.09(S D=$ 1.90).

Predicting assertiveness. The pattern of results was similar across roles in the negotiation; accordingly, the following results collapse across role. Partner ratings were used as measures of behavior (e.g., making concessions). A measure of "deal term" was created by taking the $z$ score of the settlement price across all participants and then multiplying this term by -1 for buyers (i.e., higher for sellers who sold for comparatively high prices and higher for buyers who bought for comparatively low prices).

As shown in Table 4, social expectancies were significantly related to deal terms, but instrumental expectancies were not, nor were any other measures. In contrast to this pattern, instrumental expectancies were significantly related to several ratings made by partners (including target assertiveness, overassertiveness, and competitive behavior, as well as partner trust for target and partner interaction attitudes), but social expectancies were not.

Expectancies were correlated with several of the other measures (see Table 4). Both social and instrumental expectancies were positively correlated with the TKI competitive orientation and negatively correlated with the TKI avoiding orientation. Instrumental expectancies, but not social expectancies, were correlated with TKI accommodating and NEO Assertiveness and Warmth. Expectancies were not correlated with unmitigated communion or social value items (care about results, important to get along).

A number of the nonexpectancy measures were correlated with partner ratings. TKI competitive orientation was positively correlated with partner ratings of participant competitive behavior and negatively correlated with partner trust and interaction attitudes. TKI avoiding was negatively correlated with partner ratings of participant assertiveness and positively correlated with partner interaction attitudes. NEO Assertiveness was positively related with partner ratings of participant assertiveness and negatively correlated with partner trust and interaction attitudes. NEO Angry Hostility was negatively correlated with interaction attitudes and

\footnotetext{
${ }^{2}$ In general, expectancies for low and medium assertiveness were not predictive. For instance, social expectancies for low and medium assertiveness were not significantly correlated with concession behavior, $r(59)=-.03, p=.82$; and, $r(59)=-.04, p=.78$, respectively, or with deal terms, $r(59)=-.01, p=.93$; and, $r(59)=.06, p=.63$, respectively. Likewise, instrumental expectancies for low assertiveness were not significantly correlated with concession behavior, $r(59)=.00, p=.98$; and, $r(59)=-.25, p=.78$, respectively, though instrumental expectancies for medium assertiveness were directionally correlated with concession behavior, $r(59)=-.25, p=.06$. Instrumental expectancies for low and medium assertiveness were not significantly correlated with deal terms, $r(59)=$ $-.01, p=.97$; and, $r(59)=-.07, p=.56$, respectively. As shown in Table 4 , high assertiveness expectancies were more predictive.
} 


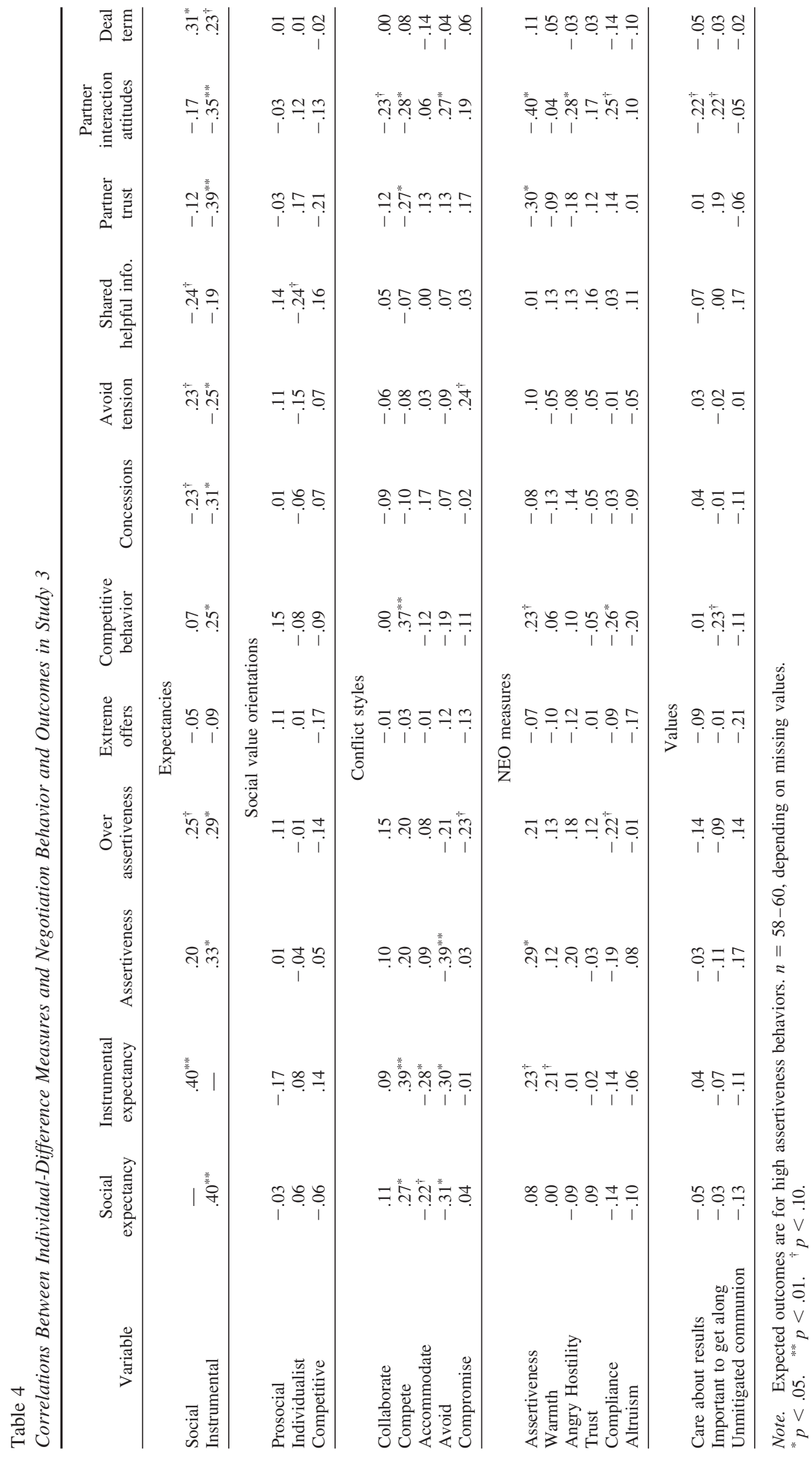


NEO Compliance was negatively correlated with partner ratings of participant competitive behavior.

Independent effect prediction. To test the independent effect prediction-that expectancies would remain predictive after controlling for the effects of values-regression models were run in cases in which both expectancy and value measures were predictive or were correlated with one another. In a model predicting partner ratings of participant assertiveness with the instrumental expectancy measure and the TKI constructs for competing, accommodating, and avoiding (i.e., the TKI measures that were correlated with instrumental expectancy), instrumental expectancies remained predictive of assertiveness $(\beta=.27), t(54)=2.07, p<$ .05 . Of the TKI measures, avoiding remained predictive $(\beta=$ -.33), $t(54)=2.13, p<.05$, whereas the others did not $(p s>$ .18). In a combined model predicting partner ratings of participant assertiveness, instrumental expectancies remained predictive $(\beta=$ $.28), t(56)=2.23, p<.05$, whereas NEO Assertiveness was only marginally so $(\beta=.23), t(56)=1.79, p=.08$.

Similar models were run predicting partner ratings of target competitive behavior, partner trust, and partner interaction attitudes. Across these models, after controlling for NEO and TKI measures, as well as SVOs and value measures, instrumental expectancies remained predictive. In summary, the independent effect prediction was generally supported. In addition, a wide range of models were analyzed to test for possible interactions between various value measures and expectancies (e.g., the interaction of social expectancies and unmitigated communion). No significant interactions emerged.

\section{Discussion}

Study 3 supported the behavioral prediction by linking expectancies to actual interpersonal behavior and outcomes. In a faceto-face zero-sum role play negotiation, those who expected more positive social outcomes at high levels of assertiveness achieved better deal terms. Those who expected more positive instrumental outcomes at high levels of assertiveness were seen as behaving more assertively. It is worth noting that the measures of participants' assertiveness were not self-reports (as in Studies 1 and 2) but rather were appraisals provided by randomly assigned negotiation partners.

Consistent with the independent effect prediction, these effects of expectancies generally remained after controlling for relevant value constructs. Although negotiation deal term was predicted by social expectancies, it was not significantly related to any of the other constructs (including SVO, conflict resolution style, unmitigated communion, and various NEO personality measures).

\section{Study 4}

In Study 4, I sought additional evidence for the link between assertiveness expectancies and real-world behavior. One important real-world instantiation of assertiveness is the inauguration of potentially productive negotiations. As Babcock, Gelfand, Small, and Stayn (2006) recently argued, the "propensity to initiate negotiations" varies from person to person, with some people habitually bypassing opportunities to negotiate when doing so would be natural and productive, whereas others chronically inaugurate negotiations, perhaps even when they are inappropriate. In Study 4,
I used a measure of propensity to initiate negotiations that included constructs that have been linked with real-world negotiation behavior, such as the number of days since a person's most recent workplace negotiation as well as days until their anticipated next workplace negotiation (Babcock et al., 2006). It was predicted that expectancies for high assertiveness behaviors would be positively associated with propensity to initiate negotiations measures for recognition and entitlement and negatively associated with the measure of apprehension.

Study 4 also gauged real-world assertiveness in the workplace, using work colleague ratings of targets' assertiveness, including their tendency to speak up and to stand their ground in a conflict. Each participant was rated by multiple colleagues who had typically worked with the participant for several years (participants were typically 28-32 years old). This dependent variable is thus a meaningful measure of real-world assertiveness, parallel to measures that have been linked in recent research on perceptions of influence and leadership in the workplace (Ames \& Flynn, 2007).

In summary, it was expected that assertiveness expectancies (measured through responses to conflict scenarios, as in Study 3) would be predictive of the propensity to initiate negotiations and colleague-rated assertiveness measures and that these links would remain after controlling for relevant motivations.

\section{Method}

Participants. Seventy-five MBA students, with a mean age of $29.0(S D=2.6)$, took part in Study 4 during a management elective course. Thirty participants $(40.0 \%)$ were women. Fortyeight participants $(64.0 \%)$ identified themselves as Caucasian, 18 $(24.0 \%)$ identified themselves as Asian or Asian American, 4 (5.3\%) identified themselves as Latino or Hispanic, and 1 (1.3\%) identified him- or herself as African American.

Expectancy and value measures. As part of a course exercise, expectancies were measured as in Study 3, as were social outcome values, unmitigated communion, and SVOs.

Propensity to initiate negotiations. A separate exercise from the above gauged the propensity to initiate negotiations using a measure including three dimensions associated with identifying and starting negotiations (Babcock et al., 2006). Recognition measures an individual's tendency to recognize situations in which negotiation is possible (four items; e.g., "There are many things available to people, if only people asked for them"). Entitlement measures the extent to which an individual feels owed or entitled to receiving an outcome better than the status quo (three items; e.g., "I think situations should be changed to fit my desires"). Apprehension measures an individual's feelings of unrest upon initiating a negotiation (five items; e.g., "I feel anxious when I have to ask for something I want"). Items were rated on a 7-point scale ranging from 1 (strongly disagree) to 7 (strongly agree).

Workplace ratings. As part of participants' course work in the prior academic year, they solicited feedback on their behaviors from former work colleagues (individuals who had worked with the students before they began their 2-year graduate program). On average, students recruited four former colleagues (who had typically worked with the student for 2-5 years and indicated they knew the student well) who provided anonymous feedback, rating various behaviors on a 7-point scale ranging from 1 (never) to 7 (always). Assertiveness in the workplace was gauged with three 
items that reflected everyday usage of the term (see Ames \& Flynn, 2007), "S/he speaks up and shares his/her views when it is appropriate," "S/he is able to stand his/her ground in a heated conflict," and "S/he is willing to engage in constructive interpersonal confrontations."

\section{Results}

Scale means and reliabilities are shown in Table 5. The two social outcome value items were only moderately correlated, $r(74)=.32, p<.01$, and, as in Studies 2 and 3, are reported separately. As in Study 3, expectancies for the high assertiveness behaviors were substantially and consistently more predictive than those for the low or medium assertiveness behaviors. ${ }^{3}$ As such, the results focus on these measures (averaged across the two scenarios) as the operationalization of expectancies. For social outcomes at high assertiveness, mean expectancies on the 11-point scale were $5.30(S D=1.70)$; for instrumental outcomes at high assertiveness, mean expectancies were $7.22(S D=1.84)$.

Predicting propensity to initiate negotiations. Expectancies were predicted to correlate with the propensity to initiate negotiations constructs. As shown in Table 6, this was significant only in the case of entitlement. Both social and instrumental expectancies were positively correlated with entitlement. Contrary to predictions, expectancies were not significantly correlated with recognition or apprehension.

The SVO measure of prosocial orientation was also correlated with entitlement. In a model predicting entitlement with instrumental expectancies and SVO prosocial, expectancies remained significantly predictive (instrumental expectancy $\beta=.38$ ), $t(71)=$ $3.40, p<.01$, whereas SVO prosocial was directionally predictive $(\beta=-.20), t(71)=-1.79, p=.08$. In a model predicting entitlement with social expectancies and SVO prosocial, expectancies were significantly predictive (social expectancy $\beta=.27$ ), $t(71)=2.37, p<.05$, as was SVO prosocial $(\beta=-.27), t(71)=$ 2.34, $p<.05$.

Predicting workplace assertiveness. Social expectancies were significantly correlated with workplace assertiveness (see Table 6). The more people expected positive social outcomes for highly assertive behaviors in a series of fictional scenarios, the more their former work colleagues saw them as assertive in the workplace, consistent with the behavioral prediction. Instrumental expectancies were not significantly correlated with workplace assertiveness.

Table 5

Scale Means and Reliabilities in Study 4

\begin{tabular}{lcccc}
\hline \multicolumn{1}{c}{ Construct } & Items & $M$ & $S D$ & $\alpha$ \\
\hline Unmitigated communion & 5 & 3.33 & 0.66 & .64 \\
SVO Prosocial & - & 3.39 & 3.79 & - \\
SVO Individualist & - & 4.83 & 3.91 & - \\
SVO Competitive & - & 0.79 & 2.35 & - \\
Apprehension & 5 & 3.96 & 1.57 & .93 \\
Recognition & 4 & 5.63 & 0.88 & .72 \\
Entitlement & 3 & 3.67 & 1.09 & .57 \\
Work assertiveness & 3 & 5.64 & 0.56 & .85 \\
\hline
\end{tabular}

Note. $\quad \mathrm{SVO}=$ Social Value Orientation; dashes represent scales where the number of items is not necessarily meaningful.
The value measures were generally unrelated to coworkers' ratings of participants' assertiveness in the workplace (see Table 6). Apprehension was negatively correlated with assertiveness. Although not necessarily a value measure, it is worth examining whether the apprehension measure usurped the predictive power of expectancies. A regression model was run, featuring both social expectancies and apprehension predicting workplace assertiveness. Both expectancies $(\beta=.33), t(67)=2.89, p<.01$, and apprehension $(\beta=-.21), t(67)=-1.87, p=.07$, were predictive in the expected directions. These results are consistent with the independent effect prediction. A wide range of models were analyzed to test for possible interactions between various value measures and expectancies. As in Study 3, no significant interactions emerged.

\section{Discussion}

In Study 4, work colleagues of participants reported their perceptions of the participants' assertiveness in the workplace. This measure of real-world assertiveness was predicted by targets' social expectancies on the basis of responses to scenarios featuring interpersonal conflicts. The separation in time and context of these measures bears reiteration: Work colleagues provided assertiveness ratings of targets typically based on several years' worth of acquaintanceship in a work context, and this measure was predicted by targets' self-reported expectancies on the basis of fictional conflict scenarios over 1 year after having left the workplace. This expectancy-behavior link remained after controlling for motives, including unmitigated communion and SVOs.

Participants also reported their propensity to initiate negotiations, components of which have been linked to real-world negotiation behavior. The measure of entitlement showed a link to expectancies, though measures of recognition and apprehension did not. The link between entitlement and expectancies remained after controlling for other measures, including SVO.

\section{General Discussion}

Why do some people come on strong in interpersonal conflicts, whereas others more readily capitulate? The traditional answer in much of the literature on conflict and negotiation revolves around interpersonal and social values. In this article, I have attempted to broaden the answer by invoking another construct psychologists have long turned to in explanations of behavior: expectancies. Four studies shed light on the form of assertiveness expectancies and on their links with behavior. In Study 1, a line drawing paradigm was

\footnotetext{
${ }^{3}$ As in Study 3, expectancies for low and medium assertiveness were generally not as predictive as expectancies for high assertiveness behaviors. For instance, social expectancies for low and medium assertiveness were not significantly correlated with entitlement, $r(74)=-.08, p=.51$; and, $r(74)=-.12, p=.32$, respectively, or with workplace assertiveness, $r(74)=.19, p=.12$; and, $r(74)=.08, p=.51$, respectively. Instrumental expectancies for medium assertiveness were not significantly correlated with entitlement, $r(74)=-.01, p=.96$, though instrumental expectancies for low assertiveness were directionally correlated with entitlement, $r(74)=-.19, p=.10$. Instrumental expectancies for low and medium assertiveness were not significantly correlated with workplace assertiveness, $r(74)=.09, p=.48$; and, $r(74)=-.07, p=.57$, respectively. As shown in Table 6 , high assertiveness expectancies were predictive.
} 
Table 6

Correlations Between Expectancies, Values, Propensity to Initiate Negotiations, and Workplace Assertiveness in Study 4

\begin{tabular}{|c|c|c|c|c|c|c|c|c|c|c|c|c|}
\hline & 1 & 2 & 3 & 4 & 5 & 6 & 7 & 8 & 9 & 10 & 11 & 12 \\
\hline 1. Social expectancies & - & & & & & & & & & & & \\
\hline 2. Instrumental expectancies & $.38^{* * *}$ & - & & & & & & & & & & \\
\hline 3. Care about results & -.05 & .03 & - & & & & & & & & & \\
\hline 4. Important to get along & .02 & .08 & $-.32^{* *}$ & - & & & & & & & & \\
\hline 5. Unmitigated communion & -.15 & -.09 & -.18 & .03 & - & & & & & & & \\
\hline 6. SVO Prosocial & .05 & -.14 & $-.38^{* * *}$ & $.20^{\dagger}$ & $.23^{\dagger}$ & - & & & & & & \\
\hline 7. SVO Individualist & -.01 & .06 & $.31^{* * *}$ & -.15 & $-.28 *$ & $-.81^{* *}$ & - & & & & & \\
\hline 8. SVO Competitive & -.08 & .13 & .09 & .07 & .09 & $-.26^{*}$ & $-.35^{* *}$ & - & & & & \\
\hline 9. Recognition & $.21^{\dagger}$ & .18 & .18 & $-.31^{* * *}$ & -.13 & -.14 & .07 & .11 & - & & & \\
\hline 10. Entitlement & $.23^{*}$ & $.39^{* *}$ & .00 & .06 & -.13 & $-.25^{*}$ & $.23^{\dagger}$ & .02 & $.27^{*}$ & - & & \\
\hline 11. Apprehension & -.18 & $-.20^{\dagger}$ & -.12 & $.25^{*}$ & .11 & -.14 & .18 & -.07 & $-.42^{* *}$ & .04 & - & \\
\hline 12. Work assertiveness & $.37^{* * *}$ & .11 & -.06 & -.19 & -.19 & .03 & .00 & -.05 & .09 & .04 & $-.28^{*}$ & - \\
\hline
\end{tabular}

Note. $n=74$ except for correlations with Work assertiveness, where $n=70$ because of missing cases. SVO $=$ Social Value Orientation.

${ }^{*} p<.05 . \quad{ }^{* * *} p<.01 .{ }^{\dagger} p<.10$.

used and revealed that most participants expected a curvilinear, inverted-U-shaped, link between assertiveness and outcomes. That is, they thought they could push up to point, but no further without incurring costs. This effect was consistent across both social and instrumental domains and for both undergraduate student and MBA student populations. This curvilinear pattern also echoes evidence of the actual impact of interpersonal assertiveness provided by Ames and Flynn (2007). However, although many participants displayed the same overall curved shape for their expectancies, considerable individual variance emerged in the parameters of the expectancies, such as the perceived optimal level of assertiveness. A link between the variance in social expectancies and self-reported assertiveness was shown in Study 1. More important, variance in expectancies was not closely related to variance in values (e.g., concern with relationships), and the link between expectancies and self-reported assertiveness remained after controlling for values.

This pattern extended across the remaining studies, which showed that expectancies had a distinct ability to predict assertive choices in a series of conflict scenarios (Study 2) and attitudes related to initiating real-world negotiations (Study 4). Measures of actual behaviors likewise showed the predicted effects: Expectancies predicted partner ratings of behavior, as well as deal terms, in a face-to-face dyadic negotiation (Study 3). Expectancies also predicted work colleague ratings of participants' assertiveness in the workplace (Study 4). Both the negotiation and workplace studies featured links between targets' self-reported expectancies and other people's perceptions of the targets' assertiveness. In short, across multiple samples and multiple measures of assertiveness, expectancies played a noteworthy predictive role that was distinct from values, including measures such as SVOs, unmitigated communion, and conflict styles. The present results suggest that expectancies can help researchers expand their answer to the question of why some people push harder than others.

None of this is to imply, of course, that values or motivations do not matter or do not help to explain assertiveness. On the contrary, the analyses reported here tended to highlight simultaneous effects for both expectancies and values. Hybrid models may best predict assertiveness by capitalizing on what social cognitive theories of personality have shown: Behavior flows from perceivers taking action on their goals in light of their idiosyncratic perceptions of circumstances (e.g., Mischel \& Shoda, 1995).

The present results did not show evidence of interactions between expectancies and values. It stands to reason that expectancies about highly valued outcomes (relational, instrumental, or other) will predict behavior more than expectancies about unvalued outcomes. The present work may not have revealed these effects because the social and relational outcomes in question were at least reasonably valuable to more or less all respondents (in effect, a restriction of range in value). Perhaps future work will uncover value-expectancy interactions through more focused measurement or situations featuring wider variance in desired outcomes.

\section{Lessons for Studying Assertiveness Expectancies}

The present results are hardly the final word on assertiveness expectancies, though they seem to suggest some directions for researchers on several different topics, such as how to measure expectancies effectively. The studies reported here revealed the potential for operationalizing assertiveness expectancies through expected outcomes for highly assertive behaviors. But would expectancies for even more assertive behavior be even more predictive? Probably not. The high assertive behaviors featured in this article (e.g., in Study 2) are forceful, but not so extreme or pathological that the participant population overwhelmingly rejected them as outrageous. At noticeably higher levels of assertion (e.g., outrageous demands, belligerence), it is likely that perceivers would increasingly share negative expectancies, and thus such expectancies would likely not be diagnostic of perceived optimal assertiveness or predictive of assertive behavior. For high assertiveness expectancies to be predictive, the behaviors they reflect need to be assertive enough to repel some individuals but not so assertive that they repulse virtually everyone.

Why would high assertiveness expectancies be especially predictive? A basic answer is that between-person variance may be greater for high assertiveness expectancies than low assertiveness ones. People may generally agree that low assertiveness approaches often fail and that moderate assertive approaches are reasonably effective; they may disagree most strikingly about what 
happens when one pushes hard. Yet, why would they disagree? It could be that aggressive and accommodating people have markedly different evidence about the outcomes of high assertiveness. Unassertive individuals might avoid assertive behavior and thus never receive clear evidence about the consequences that follow, being left instead to "catastrophize" what would have happened had they pushed harder (e.g., Vasey \& Borkovec, 1992). Assertive individuals, howwever, might engage in aggression but selectively interpret the apparent subsequent evidence, convincing themselves that their action was effective or even necessary. This asymmetry might not exist at the other end of the behavioral spectrum: Both low- and high-assertive individuals may find the disappointing or dismal outcomes obvious when they capitulate or yield. Thus, expectancies and experience could reinforce one another at high levels of assertiveness, more so than at low levels, leading more and less assertive individuals to diverge and sustain potentially exaggerated perceptions.

Both social and instrumental expectancies were gauged in all four of the studies reported here. Although social and instrumental measures tended to be moderately correlated with each other, they showed somewhat different abilities to predict other measures. In Study 1, social expectancies predicted self-rated assertiveness, though instrumental expectancies did not. In Study 2, both social and instrumental expectancies were predictive of behavioral preferences. In Study 3, social expectancies were predictive of deal terms, whereas instrumental expectancies were predictive of behaviors and partner perceptions. In Study 4, social expectancies predicted work assertiveness, whereas instrumental expectancies did not. On balance, one might conclude that social expectancies performed slightly better as a predictor. There may be psychological reasons for this (e.g., most people are generally very sensitive to social outcomes, or at least their perceptions of them) as well as measurement reasons (e.g., how these constructs were operationalized in the present studies). In order to learn more about the nature and impact of expectancies, it seems important to measure them in both the social and instrumental domains. Researchers might also attend to individual differences that moderate which expectancy is more or less predictive of behavior for different individuals.

The ability of expectancies to predict behavior naturally raises another question: What predicts - that is, generates or shapesexpectancies? It seems likely that evidence and feedback play some role in updating expectancies. Expectancies also likely have some deeper core tracing back to basic working models for the self, others, and relationships that are built up over the course of a lifetime (e.g., Campbell, Simpson, Boldry, \& Kashy, 2005). Baldwin and Keelan (1999) argued that individuals higher in trait self-esteem had more positive interpersonal expectancies about their own ability to secure affiliation from others. An exploratory analysis of the present results echoes this, showing some evidence that those lower in self-esteem were more pessimistic about their ability to pursue their interests without suffering relational costs. ${ }^{4}$ Future research could further examine these connections with working models for the self and others, revealing more about where assertiveness expectancies come from and, as a result, how they operate and evolve.

\section{Conclusion}

The present work brings together two vigorous and noteworthy traditions of research: one committed to explaining why people act with a given level of assertiveness in conflicts and negotiations and another invoking interpersonal expectancies as predictors of behavior and relations. The combination has substantial promise. The expectancies examined here showed a meaningful ability to predict assertive behavior in interpersonal conflict, helping to round out researchers' understanding of who pushes hard or holds back, and why. These expectations arguably deserve a place alongside motivations in psychologist's models and, arguably, our interventions.

\footnotetext{
${ }^{4}$ Although not a focus of the present research, self-esteem was measured in a subsample of Study 1: The MBA participants completed the Single Item Self-Esteem measure (Robins, Hendin, \& Trzesniewski, 2001). This was correlated with the social optimum measure of assertiveness at $r(101)=.17, p=.09$. The correlation with the instrumental optimum measure was not significant.
}

\section{References}

Amanatullah, E. T. (2008). Negotiating assertively: Exploring benefits of representing another to women in salary negotiations. Manuscript in preparation.

Amanatullah, E. T., Morris, M. W., \& Curhan, J. R. (in press). Negotiators who give too much: Unmitigated communion, relational anxieties, and economic costs in distributive and integrative bargaining. Journal of Personality and Social Psychology.

Ames, D. R., \& Flynn, F. J. (2007). What breaks a leader: The curvilinear relation between assertiveness and leadership. Journal of Personality and Social Psychology, 92, 307-324.

Babcock, L., Gelfand, M., Small, D., \& Stayn, H. (2006). Gender differences in the propensity to initiate negotiations. In D. De Cremer, M. Zeelenberg, \& J. K. Murnighan (Eds.), Social psychology and economics (pp. 239-259). Mahwah, NJ: Erlbaum.

Baldwin, M. W., \& Dandeneau, S. D. (2005). Understanding and modifying the relational schemas underlying insecurity. In M. Baldwin (Ed.), Interpersonal cognition (pp. 33-61). New York: Guilford Press.

Baldwin, M. W., \& Keelan, J. P. R. (1999). Interpersonal expectations as a function of self-esteem and sex. Journal of Social and Personal Relationships, 16, 822-833.

Bandura, A. (1977). Self-efficacy: Toward a unifying theory of behavioral change. Psychological Review, 84, 191-215.

Bandura, A. (1986). Social foundations of thought and action: A social cognitive theory. Englewood Cliffs, NJ: Prentice Hall.

Baumeister, R. F., \& Leary, M. R. (1995). The need to belong: Desire for interpersonal attachments as a fundamental human motivation. Psychological Bulletin, 117, 497-529.

Bowles, H. R., Babcock, L., \& Lai, L. (2007). Social incentives for gender differences in the propensity to initiate negotiation: Sometimes it does hurt to ask. Organizational Behavior and Human Decision Processes, 103, 84-103.

Campbell, L., Simpson, J. A., Boldry, J. G., \& Kashy, D. (2005). Perceptions of conflict and support in romantic relationships: The role of attachment anxiety. Journal of Personality and Social Psychology, 88, $510-531$.

Carnevale, P. J., \& De Dreu, C. K. W. (2006). Motive: The negotiator's raison d'être. In L. Thompson (Ed.), Frontiers of social psychology: Negotiation theory and research (pp. 55-76). New York: Psychology Press.

Carnevale, P. J., \& Pruitt, D. G. (1992). Negotiation and mediation. Annual Review of Psychology, 43, 531-582.

Costa, P. T., Jr., \& McCrae, R. R. (1992). The Revised NEO Personality Inventory (NEO-PIR) and NEO Five-Factor Inventory (NEO-FFI) professional manual. Odessa, FL: Psychological Assessment Resources. 
De Cremer, D., \& Leonardelli, G. J. (2003). Cooperation in social dilemmas and the need to belong: The moderating effect of group size. Group Dynamics: Theory, Research, and Practice, 7, 168-174.

De Dreu, C. K., \& Van Lange, P. A. (1995). Impact of social value orientation on negotiator cognition and behavior. Personality and Social Psychology Bulletin, 21, 1178-1188.

De Dreu, C. K., Weingart, L. R., \& Kwon, S. (2000). Influence of social motives on integrative negotiation: A meta-analytic review and test of two theories. Journal of Personality and Social Psychology, 78, 889-905.

Downey, G., \& Feldman, S. I. (1996). Implications of rejection sensitivity for intimate relationships. Journal of Personality and Social Psychology, 70, 1327-1343

Fehr, B., Baldwin, M., Collins, L., Patterson, S., \& Benditt, R. (1999). Anger in close relationships: An interpersonal script analysis. Personality and Social Psychology Bulletin, 25, 299-312.

Fritz, H. L., \& Helgeson, V. S. (1998). Distinctions of unmitigated communion from communion: Self-neglect and overinvolvement with others. Journal of Personality and Social Psychology, 75, 121-140.

Kammrath, L. K., \& Dweck, C. (2006). Voicing conflict: Preferred conflict strategies among incremental and entity theorists. Personality and Social Psychology Bulletin, 32, 1497-1508.

Komorita, S. S., \& Parks, C. D. (1996). Social dilemmas. Boulder, CO: Westview Press.

Locke, K. D., \& Sadler, P. (2007). Self-efficacy, values, and complementarity in dyadic interactions: Integrating interpersonal and social-cognitive theory. Personality and Social Psychology Bulletin, 33, 94-109.

McClintock, C. G., \& Liebrand, W. B. (1988). Role of interdependence structure, individual value orientation, and another's strategy in social decision making: A transformational analysis. Journal of Personality and Social Psychology, 55, 396-409.

Messick, D. M., \& McClintock, C. G. (1968). Motivational basis of choice in experimental games. Journal of Experimental Social Psychology, 3, $85-101$
Mischel, W. (1973). Toward a cognitive social learning reconceptualization of personality. Psychological Review, 80, 252-283.

Mischel, W., \& Shoda, Y. (1995). A cognitive-affective system theory of personality: Reconceptualizing situations, dispositions, dynamics, and invariance in personality structure. Psychological Review, 102, 246-268.

Olekalns, M., \& Smith, P. L. (2003). Testing the relationships among negotiators' motivational orientations, strategy choices and outcomes. Journal of Experimental Social Psychology, 39, 101-117.

Raudenbush, S., Bryk, A., Cheong, Y. F., \& Congdon, R. (2004). HLM 6: Hierarchical linear and nonlinear modeling. Lincolnwood, IL: Scientific Software International.

Robins, R. W., Hendin, H. M., \& Trzesniewski, K. H. (2001). Measuring global self-esteem: Construct validation of a single-item measure and the Rosenberg Self-Esteem Scale. Personality and Social Psychology Bulletin, 27, 151-161.

Roese, N. J., \& Sherman, J. W. (2007). Expectancy. In A. W. Kruglanski \& E. T. Higgins (Eds.), Social psychology: A handbook of basic principles (Vol. 2, pp. 91-115). New York: Guilford Press.

Thomas, K. W., \& Kilmann, R. H. (2002). Thomas-Kilmann Conflict Mode Instrument. Palo Alto, CA: CPP, Inc.

Tolman, E. C. (1932). Purposive behavior in animals and men. New York: Appleton-Century-Crofts.

Van Lange, P. A. M. (1999). The pursuit of joint outcomes and equality in outcomes: An integrative model of social value orientation. Journal of Personality and Social Psychology, 77, 337-349.

Van Lange, P. A. M., Otten, W., De Bruin, E. M. N., \& Joireman, J. A. (1997). Development of prosocial, individualistic, and competitive orientations: Theory and preliminary evidence, Journal of Personality and Social Psychology, 73, 733-746.

Vasey, M., \& Borkovec, T. D. (1992). A catastrophizing assessment of worrisome thoughts. Cognitive Therapy \& Research, 16, 505-520.

White, J. B., Tynan, R., Galinsky, A. D., \& Thompson, L. (2004). Face threat sensitivity in negotiation: Roadblock to agreement and joint gain. Organizational Behavior and Human Decision Processes, 94, 102-124.

Appendix

Scenarios and Endings for Studies 2, 3, and 4

\section{Salary Negotiation}

Scenario beginning: Imagine that you are negotiating with a potential employer about the terms of their job offer to you. The manager you'd be working with calls you on the phone with the offer, including a salary of $\$ 92,000$. This figure might be acceptable to you but is noticeably below what you know similar people make in similar positions $(\$ 110,000-\$ 120,000)$. The job is your top choice, but you were expecting a considerably higher offer. You are fairly sure there is room for negotiation. The manager asks you if you're ready to accept.

High assertiveness behavior: You make a counteroffer of $\$ 120,000$.

Medium assertiveness behavior: You make a counteroffer of $\$ 105,000$.

Low assertiveness behavior: You accept the existing $\$ 92,000$ offer without further negotiation.

\section{Managing Freelancer}

Scenario beginning: Imagine that you are working in a consulting firm and are preparing a series of company profiles for an important client. A freelance graphics artist who you work with regularly (you're his major customer, and he's your most effective freelancer) has been taking the raw text and figures you've been sending by e-mail and creating the presentation-ready profile documents. Each one takes about an hour, and the freelancer charges you $\$ 80$ an hour (\$80 per profile). The freelancer sent you what he thought was the final profile and said he was planning to take a few much-needed days off. But you've just realized there are 10 more profiles to do. You don't know what happened, or who, if anyone, is to blame, but the e-mail you sent to him with this final batch didn't get through. You have 24 hours before everything needs to be ready. It's possible that your company's internal graphics artists could help, but they are often slow and unreliable.

High assertiveness behavior: You thank the freelancer and explain the situation. You tell him that without additional evidence, you can't be sure this wasn't his mistake. You remind him that you are his major customer and that you expect his flexibility and support under deadlines. You tell him that you need the 10 profiles done in the next day and that you'll pay the regular rate $(\$ 800)$. 
Medium assertiveness behavior: You thank the freelancer for his work and explain the situation to him. You tell him you'd be grateful if he could help out in any way. You offer to pay him for 10 hours (\$800) if he could do just five of the profiles (normally 5 hours of work).

Low assertiveness behavior: You thank the freelancer for his work and tell him you hope he has a good break. You begin to contact your internal graphics department to see if they can help.

\section{Team Meeting}

Scenario beginning: Imagine that you're in a team meeting with four colleagues on a strategic planning committee in a mid-sized manufacturing organization. It's the first meeting of the team, and you don't know the other team members that well. The discussion is revolving around potential new markets to enter, and one of the team members begins to strongly advocate entering a new country with your current products. He goes on for a few minutes very energetically and then concludes, "This is a huge opportunity just waiting for us. We'd be idiots to not pursue this." A year ago, you and a subordinate did a quick analysis of this same country's market. Although the market appeared to be sizable and growing somewhat quickly, you discovered that the regulatory hurdles were significant and that the competitive response would be considerable. You also saw major challenges in arranging distribution. You concluded that short-term success would be difficult, and there was a real potential for wasting considerable resources better invested elsewhere. Your teammate just finished advocating for the market, reiterating with intensity, "Seriously, we' $\mathrm{d}$ be fools to let this slip away."

High assertiveness behavior: You say, "I totally disagree. This market is a complete dead-end for us and a potentially huge waste of time and resources. Believe me, I've already looked it over. There are dozens of other better uses for our money. You won't get me to sign off on any strategic plan that includes this market. We should focus this committee's attention elsewhere."

Medium assertiveness behavior: You say, "I can see why this market could look attractive from the outside, especially given its growth. A while back, I looked into this opportunity and I'd be happy to share the details of this research with you. But my recollection is that we identified multiple barriers that made this opportunity look unattractive. I welcome everyone's opinion on this, but I think we should proceed with caution."

Low assertiveness behavior: You hold back and say nothing, allowing the conversation to continue, hoping that with further analysis, the truth about the market will come out and that it won't be attributed to you.
Received December 27, 2006

Revision received June 16, 2008

Accepted June 27, 2008 